\title{
Psychological stress downregulates epidermal antimicrobial peptide expression and increases severity of cutaneous infections in mice
}

\author{
Karin M. Aberg, ${ }^{1}$ Katherine A. Radek, ${ }^{2}$ Eung-Ho Choi, ${ }^{3}$ Dong-Kun Kim, ${ }^{3}$ Marianne Demerjian, ${ }^{1}$ \\ Melanie Hupe, ${ }^{1}$ Joseph Kerbleski, ${ }^{1}$ Richard L. Gallo, ${ }^{2}$ Tomas Ganz, ${ }^{4}$ Theodora Mauro, ${ }^{1}$ \\ Kenneth R. Feingold, ${ }^{1}$ and Peter M. Elias'1
}

1Dermatology and Medical (Metabolism) Services, Veterans Affairs Medical Center, Department of Dermatology, and Department of Medicine, UCSF, San Francisco, California, USA. ${ }^{2}$ Dermatology Service, Veterans Affairs Medical Center, and Department of Dermatology, UCSD, La Jolla, California, USA. ${ }^{3}$ Department of Dermatology, Yonsei University, Wonju, Republic of Korea. ${ }^{4}$ Division of Pulmonary, Critical Care Medicine, and Hospitalists, UCLA David Geffen School of Medicine, Los Angeles, California, USA.

\begin{abstract}
The skin is the first line of defense against microbial infection, and psychological stress (PS) has been shown to have adverse effects on cutaneous barrier function. Here we show that PS increased the severity of group A Streptococcus pyogenes (GAS) cutaneous skin infection in mice; this was accompanied by increased production of endogenous glucocorticoids (GCs), which inhibited epidermal lipid synthesis and decreased lamellar body (LB) secretion. LBs encapsulate antimicrobial peptides (AMPs), and PS or systemic or topical GC administration downregulated epidermal expression of murine AMPs cathelin-related AMP and $\beta$-defensin 3. Pharmacological blockade of the stress hormone corticotrophin-releasing factor or of peripheral GC action, as well as topical administration of physiologic lipids, normalized epidermal AMP levels and delivery to LBs and decreased the severity of GAS infection during PS. Our results show that PS decreases the levels of 2 key AMPs in the epidermis and their delivery into LBs and that this is attributable to increased endogenous GC production. These data suggest that GC blockade and/or topical lipid administration could normalize cutaneous antimicrobial defense during PS or GC increase. We believe this to be the first mechanistic link between PS and increased susceptibility to infection by microbial pathogens.
\end{abstract}

\section{Introduction}

Multiple converging lines of evidence suggest that psychological stress (PS), if sustained, can adversely impact critical functions such as immune surveillance (1), gastrointestinal integrity (2-4), coronary artery disease $(5,6)$, and wound healing (7-12). Although recent studies in both humans and experimental animals suggest that PS compromises host defenses against bacterial and viral infections (13-21), the pathogenic mechanisms remain unknown. Three potentially interconnected mechanisms have been proposed to explain the negative impact of PS on host defenses against infection and neoplasia: (a) psychoneuroimmunoendocrine dysfunction, which leads to increased proinflammatory neuropeptide and cytokine production in a manner either dependent or independent of the hypothalamic-pituitary-adrenal (HPA) axis (18-24); (b) increased plasma levels of endogenous glucocorticoid (GC) caused by activation of the HPA axis (16, 18-21, 24, 25); and (c) a cutaneous steroidogenic system, with localized production of corticotropin-releasing factor $(\mathrm{CRF})(26,27)$, which could mediate the adverse effects of PS on skin.

Nonstandard abbreviations used: AMP, antimicrobial peptide; CRAMP, cathelinrelated AMP; CRF, corticotropin-releasing factor; GAS, group A Streptococcus pyogenes; GC, glucocorticoid; GCr, GC receptor; HPA, hypothalamic-pituitary-adrenal; LB, lamellar body; mBD, mouse $\beta$-defensin; PS, psychological stress; SC, stratum corneum.

Conflict of interest: The authors have declared that no conflict of interest exists. Citation for this article: J. Clin. Invest. 117:3339-3349 (2007). doi:10.1172/JCI31726
The negative consequences of PS on critical epithelial functions (in contrast to those of other tissues) such as epidermal permeability barrier homeostasis (28-30) could be ascribed largely - if not entirely - to a PS-induced increase in circulating levels of GCs, because blockade of either GC production by systemic administration of a CRF inhibitor or GC peripheral action with a $\mathrm{GC}$ receptor $(\mathrm{GCr})$ antagonist normalizes permeability barrier function in mice subjected to multiple forms of PS $(31,32)$. The central role of a PS-induced increase in endogenous GCs is further supported by the observation that short-term administration of either systemic or topical GCs produces an almost-identical spectrum of epidermal abnormalities (33).

The adverse effects of PS or GC increase on cutaneous permeability barrier function can be further ascribed to an inhibition of epidermal lipid synthesis, leading to decreased production of epidermal lamellar bodies (LBs) $(33,34)$, multifunctional organelles that deliver endogenous lipids, desquamatory enzymes, and antimicrobial peptides (AMPs) to the stratum corneum (SC) interstices, thereby providing for the permeability and antimicrobial barriers $(35,36)$. As noted above, LBs in human epidermis encapsulate and secrete not only lipids, but also at least 2 AMPs, $\beta$-defensin (hBD2) and the cathelicidin hCap18 carboxyterminal fragment LL-37 $(37,38)$. Because our prior studies showed that protein delivery to nascent epidermal LBs is dependent upon prior or concurrent deposition of lipids within this organelle (39), we hypothesized that PS, by inhibiting epidermal lipid synthesis and 
A

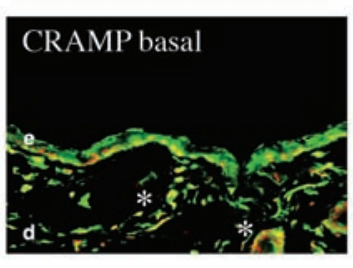

B

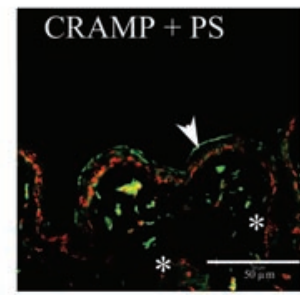

D

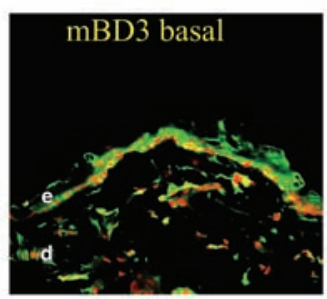

E

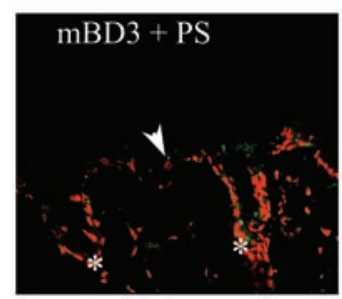

C

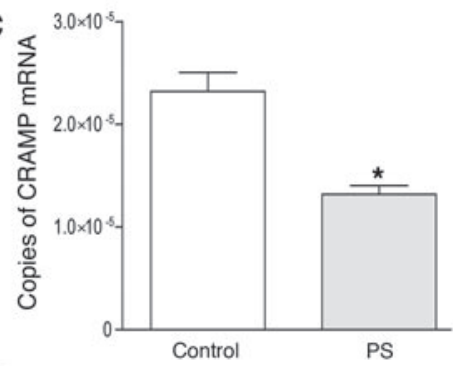

$\mathbf{F}$

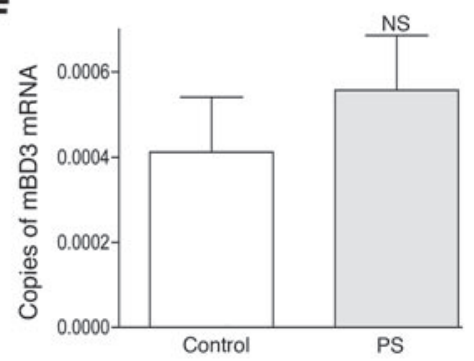

Figure 1

PS downregulates epidermal AMP expression. Normal hairless mice ( $n=3$ each for immunohistochemistry and RT-PCR studies; 3-4 replications for each experiment in these and subsequent experiments) were exposed to insomnia- and crowding-induced PS (B and $\mathbf{E}$ ) for $72 \mathrm{~h}$, while littermate controls $(\mathbf{A}$ and $\mathbf{D})$ were not stressed. Frozen sections $(8 \mu \mathrm{m})$ were stained with primary antibodies to CRAMP and mBD3 and processed as described in Methods (for controls, see Supplemental Figure 1). Throughout the figures, white arrows indicate normal levels of positive immunostaining (green); white arrowheads indicate reduced staining; asterisks indicate positive immunostaining of pilosebaceous follicles; and "d" and "e" indicate dermis and epidermis, respectively. (C and F) mRNA was extracted from PS and control mouse epidermis, followed by quantitative RT-PCR (see Methods). Normalization in this and all subsequent studies was to $18 \mathrm{~S}$ mRNA, with 2-3 replicates per sample $(n=3$ per cohort). Scale bars: $50 \mu \mathrm{m}$. ${ }^{\star} P=0.007$.

LB production in a GC-dependent manner, could prevent delivery of both these AMPs to these organelles. If PS decreases AMP bioavailability in epidermis, the link between PS/epidermal lipids and LB/AMP could then explain the apparent increased occurrence of cutaneous infections in association with PS $(14,16,17)$. We hypothesized further that if the putative link between PS and decreased AMP production is mediated by increased production of endogenous GCs and/or peripheral action of GCs, then AMP expression could be normalized by blockade of endogenous GC production or peripheral action. Finally, because the negative effects of PS or GC increase on epidermal lipid synthesis and LB production can be overcome by topical physiologic lipid replacement $(33,34)$, we hypothesized that comparable lipid replenishment could also restore production/bioavailability of one or both AMPs. Our studies determined that PS decreases levels of 2 key AMPs in the epidermis by divergent mechanisms, both ultimately mediated by an increase in endogenous GC. This decrease in AMP increased the severity of cutaneous GAS infections, and GC blockade and/or topical physiologic lipids could normalize epidermal antimicrobial defense in the face of PS/GC. Finally, we demonstrated that physiologic GC account for the low, constitutive expression of these AMP under nonstressed conditions.

\section{Results}

PS downregulates murine epidermal cathelicidin and mouse $\beta$-defen$\sin 3$ protein levels by divergent mechanisms. To ascertain whether PS modulates epidermal AMP levels, we first assessed cathelin- related AMP (CRAMP) and mouse $\beta$-defensin 3 (mBD3; the closest murine homolog of $\mathrm{hBD} 2$ ) protein levels in mice subjected to a global form of PS (i.e., combined insomnia-, noise-, and crowding-induced PS sustained for up to $72 \mathrm{~h}$; refs. 32,34 ). Both immunostainable CRAMP and $\mathrm{mBD} 3$ protein were readily detected by immunofluorescence throughout the epidermis of nonstressed control mice (Figure 1, A and D). Western immunoblotting also showed a 3- to 4-kDa band in epidermis of nonstressed normal mice under basal conditions (Supplemental Figure 1G; supplemental material available online with this article; doi:10.1172/ JCI31726DS1), further confirming the specificity of the $\mathrm{mBD} 3$ antibody. In contrast, $\mathrm{PS}$ provoked a decline in both epidermal CRAMP and mBD3 immunostainable protein (Figure 1, B and E; see Supplemental Figure 1 for controls, including immunostaining for $\mathrm{MBD} 3$ in CRAMP knockout epidermis). Moreover, AMP immunostaining declined not only in epidermis, but also to an equivalent extent in cutaneous pilosebaceous structures in PS-exposed mice (Figure 1, B and $\mathrm{E}$, asterisks). PS did not significantly alter epidermal mRNA levels for $\mathrm{mBD} 3$ (Figure 1F), although the decline in CRAMP protein correlated with decreased epidermal cathelicidin mRNA levels (Figure 1C). These results show that PS downregulated CRAMP at the mRNA and protein levels, while PS decreased $\mathrm{mBD} 3$ at the protein level only; the decline in both AMP proteins in pilosebaceous epithelia suggests that PS could also downregulate AMPs in extraepidermal epithelia.

As with PS, exogenous GCs downregulate epidermal AMP expression. To test whether the negative effects of PS can be attributed to increased endogenous GCs, we initially ascertained whether supraphysiologic doses of systemic or topical GCs downregulate epidermal AMP production. For the studies with systemic GCs, we administered 450 $\mu \mathrm{g} / \mathrm{kg}$ dexamethasone intraperitoneally to hairless mice 3 times over $72 \mathrm{~h}$, a dose previously shown to compromise epidermal differentiation, proliferation, lipid synthesis, and permeability barrier function (32-34). Whereas intraperitoneal administration of vehicle alone to normal mice (Figure 2, B and E) did not alter either CRAMP or $\mathrm{mBD} 3$ levels compared with untreated controls (Figure 2, A and D), systemic GCs markedly downregulated immunostaining for CRAMP and mBD3 protein levels both in epidermis and in pilosebaceous structures (Figure 2, C and F, asterisks).

Topical administration of the "superpotent" class I topical GC clobetasol, like systemic GCs, also alters permeability barrier homeostasis by inhibiting epidermal lipid synthesis, which leads to decreased epidermal LB production (33). Hence, we next assessed whether topical clobetasol, like systemic GCs, also alters epidermal AMP expression. Protein levels for both CRAMP and mBD3 mark- 
A

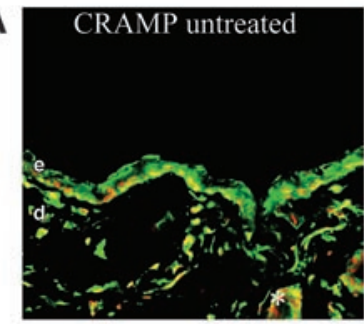

D

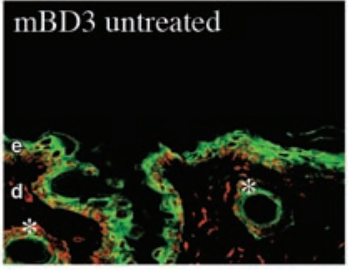

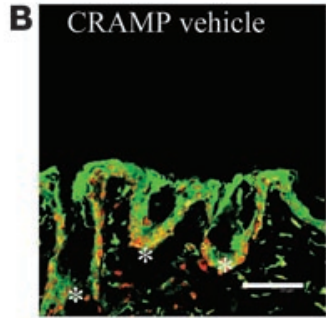

E $\mathrm{mBD} 3$ vehicle

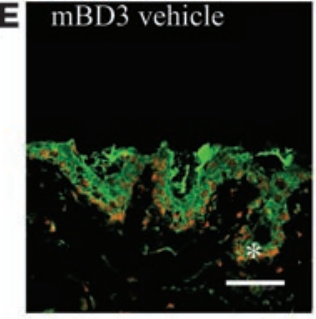

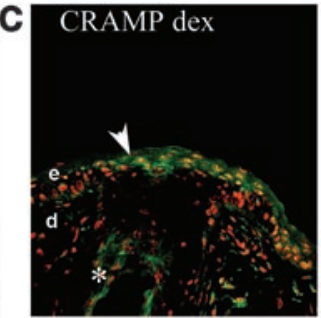

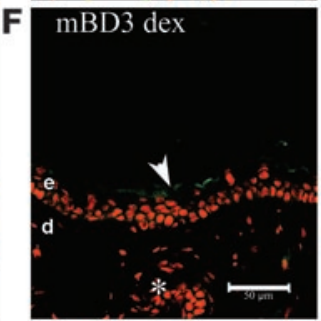

Figure 2

Systemic steroid-induced downregulation of epidermal AMPs mimics effects of PS. Cohorts of normal hairless mice ( $n=3$ per cohort) received intraperitoneal dexamethasone (dex; $450 \mu \mathrm{g} / \mathrm{cell} / \mathrm{kg}$ daily for $3 \mathrm{~d}, \mathbf{C}$ and $\mathbf{F}$ ) or vehicle alone (daily for $3 \mathrm{~d}$, $\mathbf{B}$ and $\mathbf{E})$ or were left untreated (A and $\mathbf{D})$. Biopsies were obtained for immunostaining for CRAMP and mBD3 (see Methods). Scale bar: $50 \mu \mathrm{m}$.

CRF) intraperitoneally to block GC production. Like RU-486, antalarmin cotreatment again appeared to normalize or supernormalize both mBD3 and CRAMP immunostaining in the face of ongoing PS (Figure 4, compare D and $\mathrm{H}$ with $A$ and E). Finally, antalarmin also increased AMP immunostaining in pilosebaceous structures (Figure 4, D and H, asterisks). Together, these results strongly suggest that the adverse effects

edly declined after 3 applications of topical clobetasol over a 72-h time period (Figure 3). Finally, in contrast to both PS and systemic GCs, topical GCs did not appear to reduce immunostaining for AMPs in pilosebaceous structures (Figure 3, B and D, asterisks). However, because these hairless mice have less prominent pilosebaceous ducts, it is possible that clobetasol would suppress AMP production within follicles of hairy mice.

Finally, to clarify further whether the PS-induced increase in endogenous GCs inhibits AMP expression at the mRNA or protein level, we next added dexamethasone (10 nM) to second-passage cultured human keratinocytes. Whereas keratinocyte mRNA levels for LL-37 (the cathelicidin human homolog) declined in response to dexamethasone treatment, mRNA levels for hBD2 (the human homolog of mBD3) did not change (Supplemental Figure 2). Together, these results further suggest that GCs regulate CRAMP/LL-37 mRNA and protein expression, while hBD2/mBD3 is regulated at the protein level alone.

PS-induced AMP downregulation is mediated by increased endogenous GCs. Prior studies have demonstrated that a PS-induced increase in endogenous GCs largely accounts for the negative effects of PS on epidermal structure and function $(31,32,34)$. To assess whether a PS-induced increase in endogenous GCs also accounts for the PS-induced decline in AMP levels, we first assessed whether blockade of GC peripheral action by systemic administration of the GCr inhibitor mifeprostone (RU-486) would restore epidermal AMP levels in the face of ongoing PS. PS again suppressed immunostaining for both CRAMP and $\mathrm{mBD} 3$ in epidermis (Figure 4, compare B and $\mathrm{F}$ with $\mathrm{A}$ and $\mathrm{E}$ ). Yet when PS mice were cotreated with RU-486, epidermal protein levels for both CRAMP and $\mathrm{mBD} 3$ normalized, or even appeared to increase to supernormal levels (Figure 4, C and G). Moreover, RU-486 cotreatment also normalized AMP immunostaining in pilosebaceous structures (Figure 4, C and G, asterisks).

Whereas the results of the experiment with RU-486 suggest that the PS-induced downregulation of AMP expression results from peripheral action of GCs, RU-486 exhibits broad, and sometimes unrelated, pharmacologic activities. Hence, we next employed an alternate approach to assess the role of increased GCs by determining whether increased endogenous GC production also downregulates AMP expression during PS. For these studies, during ongoing PS we coadministered antalarmin (a pharmacologic inhibitor of of PS on epidermal and pilosebaceous AMP production can be attributed to an increase in endogenous GC production and/or peripheral action.

$P S$-induced downregulation of AMP delivery to epidermal LBs is reversed by GC blockade. As described above, the human homologs of CRAMP and $\mathrm{mBD} 3$ (LL-37 and hBD2, respectively) are sequestered within epidermal LBs in preparation for their putative secretion into the SC interstices. We next used immunoelectron microscopy to ask whether PS alters the loading of mouse AMPs into nascent LBs. In mice subjected to mice, LBs not only were reduced in number (see below), but also demonstrated little or no immunolabeling for either CRAMP or mBD3 (Figure 5, A and B). Where labeling was present in PS mice, it was scattered instead at low levels throughout the cytosol of stratum granulosum cells (Figure 5, A and B, circles). In contrast, when PS mice were cotreated with RU-486, many LBs again demonstrated immunolabeling of both AMPs within their internal contents. Finally, we quantified the extent of LB immunolabeling for CRAMP and $\mathrm{MBD} 3$ in randomized, coded
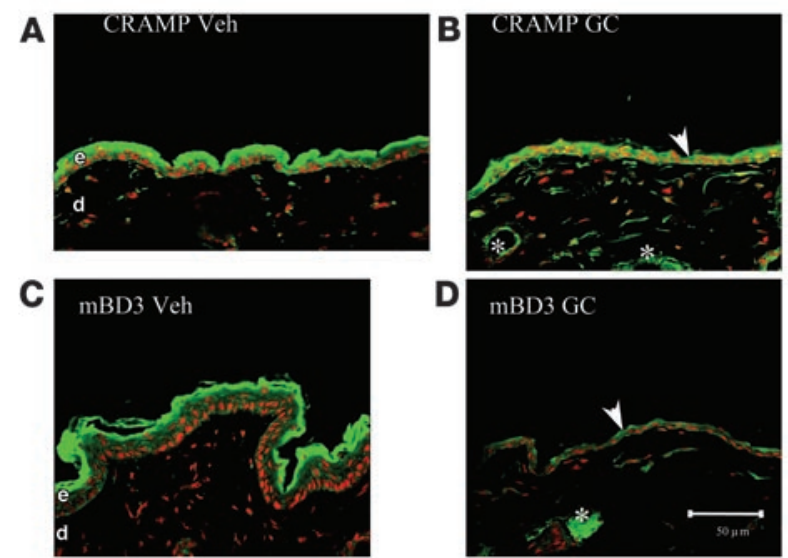

\section{Figure 3}

Superpotent topical steroid also downregulates epidermal AMPs. Normal hairless mice ( $n=3$ per group) were treated topically with either clobetasol $\left(0.05 \%\right.$, B and D) or vehicle $\left(60 \mu \mathrm{l}\right.$ to a $3-\mathrm{cm}^{2}$ area, $\mathbf{A}$ and $\mathbf{C})$ once daily for $3 \mathrm{~d}$. Frozen sections $(8 \mu \mathrm{m})$ were immunostained for either CRAMP ( $\mathbf{A}$ and $\mathbf{B}$ ) or mBD3 (C and $\mathbf{D}$ ) (see Methods). Scale bar: $50 \mu \mathrm{m}$. 

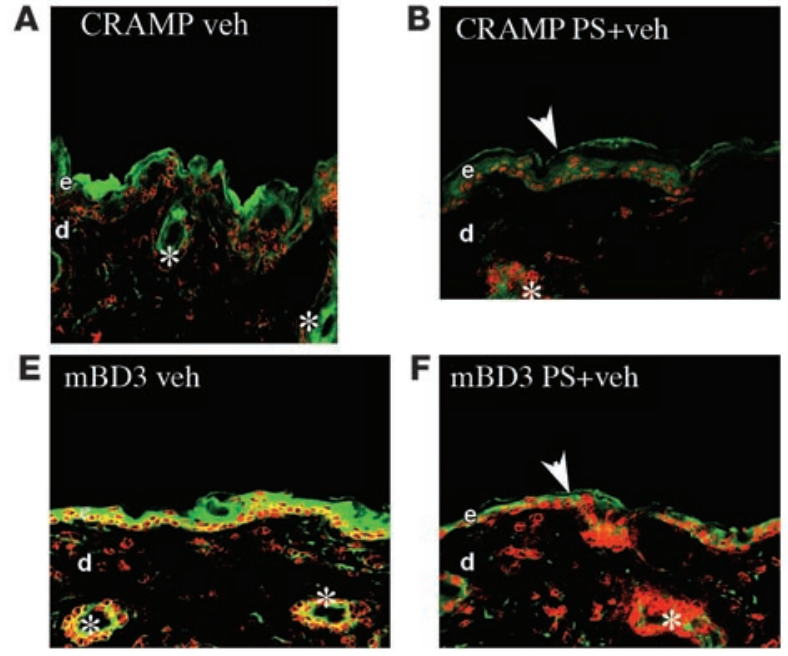
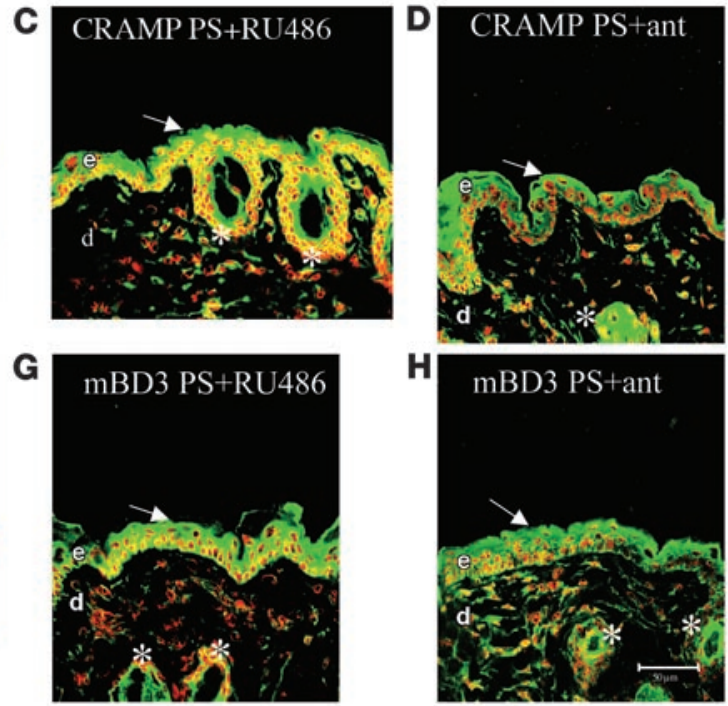

\section{Figure 4}

PS-induced downregulation of epidermal AMPs is reversed by blockade of endogenous GC production and/or action. Hairless mice $(n=3$ per group) were exposed to PS with concurrent intraperitoneal administration of either RU-486 (C and $\mathbf{G}$ ), antalarmin (ant; $\mathbf{D}$ and $\mathbf{H}$ ), or vehicle (B and F). For dosage, timing, and drug concentrations, see Methods. Frozen sections $(8 \mu \mathrm{m})$ were immunostained with CRAMP (A-D) or mBD3 (E-H) primary antibodies (see Methods). Samples A and E were from untreated control littermates. Scale bar: $50 \mu \mathrm{m}$.

micrographs from PS mice with or without RU-486 treatment as well as nonstressed mice. Labeling for both AMPs declined by over $80 \%$ in LBs of PS versus nonstressed mice, but it either normalized or became supernormal in PS mice cotreated with RU-486 (Figure $5, \mathrm{C}$ and D). Together, these results demonstrate first, by an alternate method, that PS reduces production and/or delivery of AMPs, and second, that such reduced delivery to LB reflects a PS-induced increase in endogenous GCs.

Endogenous GCs account for low constitutive levels of AMP production in normal epidermis. Because both hBD2 and cathelicidin demonstrate low, constitutive expression in normal human epidermis $(40,41)$, and because blockade of endogenous GCs in PS mice resulted in supernormal AMP immunostaining, we next asked whether the low expression of these AMPs can be attributed to physiologic levels of endogenous GCs. Indeed, both RU-486 and antalarmin treatment increased immunostaining for $\mathrm{mBD} 3$ and CRAMP in normal murine epidermis (Figure 6, A-C and E-G). Moreover, blockade in the production and/or action of endogenous GCs also increased immunostaining for both AMPs in pilosebaceous structures (Figure 6, B, C, F, and G, arrows). Furthermore, epidermal immunostaining for CRAMP and mBD3 increased markedly in adrenalectomized mice (differences for CRAMP were more striking than for mBD3; Figure 6, D, H, I, and J), as did immunolabeling of both AMPs in pilosebaceous follicles (Figure 6, D and H, arrows). Yet, despite the adverse effects of excess GCs on epidermal structure and function (see above), adrenalectomy did not improve epidermal permeability barrier homeostasis (Supplemental Figure 3). These results strongly suggest that the low constitutive expression of $\mathrm{mBD} 3$ and CRAMP in epidermis and appendages can be attributed to physiologic levels of endogenous GCs, but such physiologic levels of GCs do not alter cutaneous permeability homeostasis.

Exogenous lipids override the negative effects of PS and GCs on $m B D 3$, but not CRAMP, expression and delivery to $L B$. Topical replacement of the 3 inhibited lipids (cholesterol, ceramides, and free fatty acids) reverses PS- or GC-induced abnormalities in epidermal LB production $(33,34)$ (Supplemental Figure 4), and protein delivery to LB is dependent upon prior and/or concurrent lipid deposition within these organelles (39). Hence, we next asked whether coadministration of a mixture of ceramides, cholesterol, and free fatty acids at a 1:1:1 molar ratio, designed to correct the PS- or GC-induced inhibition of epidermal lipid synthesis $(33,34)$, normalizes AMP production in the face of PS. Both PS and GCs again downregulated $\mathrm{mBD} 3$ and CRAMP protein levels (Figure 7, A, B, D, and E, and Supplemental Figure 5). As previously described $(33,34)$, the topical lipid mixture normalized both the density (i.e., production) of LB and the LB content in the face of PS or topical GC therapy (Supplemental Figure 4). Yet, while the coapplied lipid mixture partially normalized $\mathrm{mBD} 3 \mathrm{immunolabeling} \mathrm{in} \mathrm{the} \mathrm{face} \mathrm{of} \mathrm{both} \mathrm{PS}$ and topical GCs (Figure 7, C and F), it failed to increase CRAMP immunolabeling under the same conditions (Supplemental Figure 5), effectively ruling out the possibility that the lipids could be interfering with GC uptake. Furthermore, as noted above, topical lipid treatment of PS animals increased mBD3, but not CRAMP, uptake into epidermal LBs (Figure 5, A, B, E, and F). Finally, the topical lipids did not reverse the PS-induced decline in either CRAMP or mBD3 immunostaining of pilosebaceous structures (Figure 7, C and F, arrows, and Supplemental Figure 5). These results show that topical lipid replacement partially normalized the epidermal protein levels of $\mathrm{mBD} 3$ as well as the deposition of this AMP within epidermal LBs, consistent with posttranscriptional regulation of expression of this AMP by PS or GCs. Conversely, lipid replacement increased neither CRAMP protein levels nor deposition in LB, consistent with regulation of this AMP by PS or GCs instead at a transcriptional level.

PS-induced decline in epidermal AMPs correlates with increased susceptibility to cutaneous infections and is mediated by GCs. Prior studies have shown that CRAMP knockout mice exhibit increased susceptibility to group A Streptococcus pyogenes (GAS) skin infections (42). 

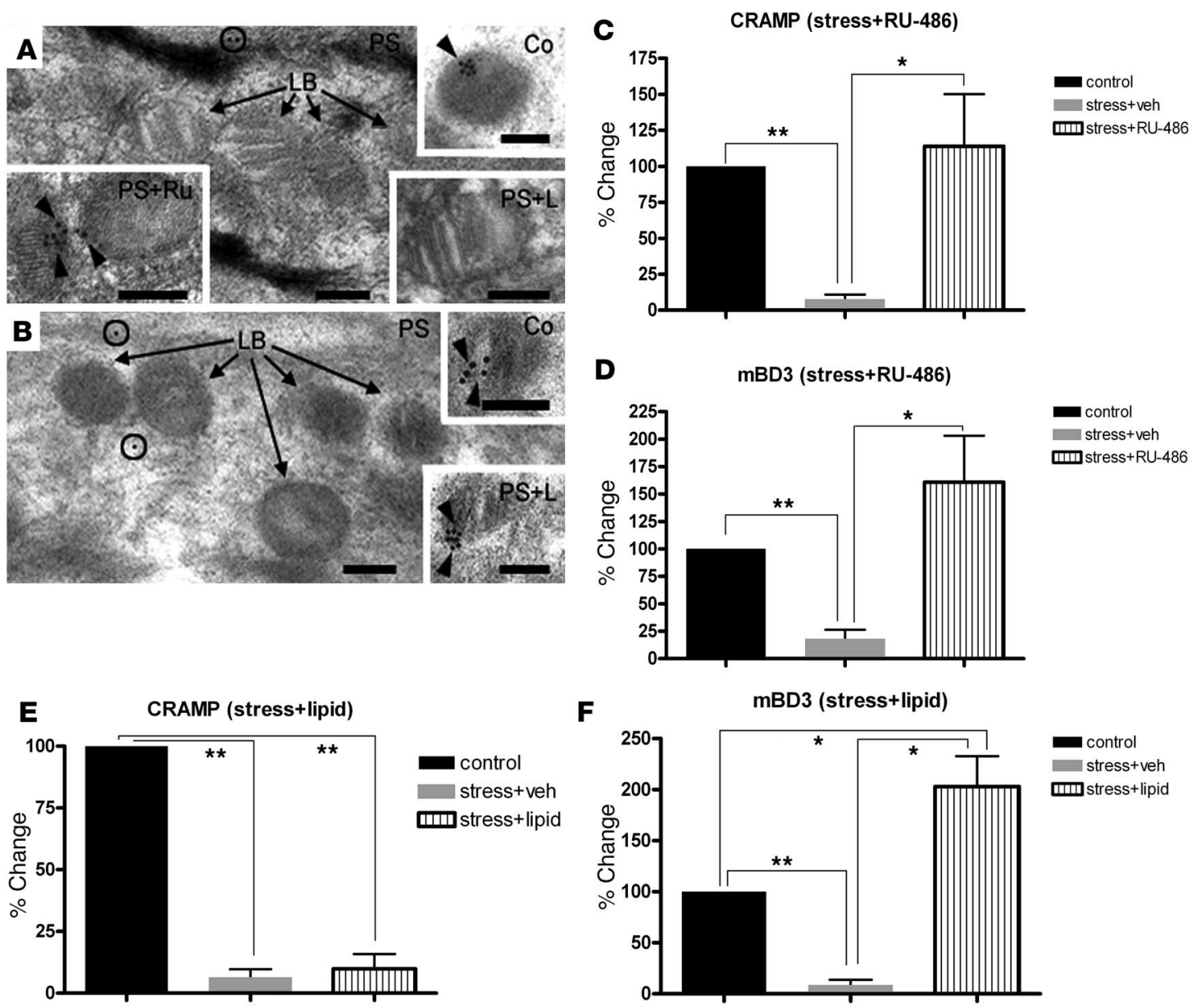

\section{Figure 5}

PS-induced decrease in AMP delivery to epidermal LBs is reversed by RU-486. (A and B) Ultrathin sections labeled with CRAMP (A) and mBD3 (B) primary antibodies followed by a 10-nm colloidal gold-tagged secondary antibody after embedding for electron microscopy. Sections were postfixed in osmium tetroxide and embedded in LR White medium. Black arrows denote unlabeled LBs; circles indicate label in cytosol. (A) CRAMP was labeled (black arrowheads) in nonstressed normal controls (Co), and CRAMP labeling reappeared in PS mice treated with RU-486 $(P S+R u)$, but not with exogenous lipids (PS+L). (B) Exogenous lipids restored labeling of mBD3 in LB in PS mice (black arrowheads). Scale bars: $100 \mathrm{~nm}$. (C-F) Quantitative data for immunolabeling of CRAMP and mBD3 in LB in nonstressed control or PS mice plus either RU-486 (C and D) or lipid (E and F) cotreatment. ${ }^{*} P<0.05 ;{ }^{*} P<0.001$.

Because PS substantially reduced CRAMP levels in both epidermis and skin appendages, we next assessed directly whether PS increases the severity of cutaneous GAS infections. In nonstressed mice, skin abscesses peaked in size at $20 \pm 10 \mathrm{~mm} 4 \mathrm{~d}$ after intracutaneous inoculation of GAS, receding to $10 \pm 3 \mathrm{~mm}$ by $5 \mathrm{~d}$ (Figure 8 ). In PS mice, mean lesion size was almost twice that of nonstressed mice, and abscesses persisted longer. In contrast, when PS mice were cotreated with RU-486, skin abscesses were comparable in size to those of nonstressed mice (Figure 8, C and D). However, RU486 did not decrease the severity of GAS infections in nonstressed mice. Together, these results demonstrate that the PS-induced, GC-mediated reduction in AMPs has negative consequences for resistance to cutaneous GAS infection.

\section{Discussion}

PS adversely effects the function of epithelial tissues, including the epidermis, where it perturbs both epidermal permeability barrier homeostasis (28-31) and cutaneous wound healing (1,
3, 7-12). Although PS-induced functional abnormalities (23, 43, 44) are often attributed to psychoneuroimmune abnormalities, PS-induced stimulation of endogenous GC production, which compromises both epidermal lipid synthesis and LB production, accounts for epidermal dysfunction (32-34). Because PS increases systemic GC levels and systemic administration of both the CRF inhibitor antalarmin and the GCr antagonist RU-486 completely normalize function, HPA-derived GCs are likely important mediators of the effects of PS on epidermal function. Yet the recently described cutaneous steroidogenic system $(26,27,45,46)$ could also be a participant, because the skin elaborates not only CRF (47, 48), but also corticosteroids, which are generated by different types of cells within the skin $(49,50)$.

We show here that PS also inhibited another key function: antimicrobial defense. Moreover, the decrease in epidermal AMP production was sufficient to increase the severity of infections from at least one important bacterial pathogen, GAS. Thus, the PS-induced decline in AMPs could account for the association between PS and 
A

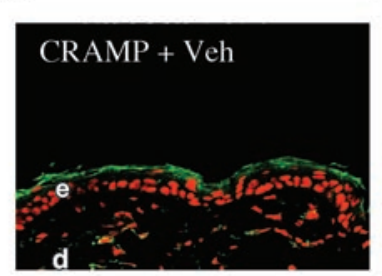

E

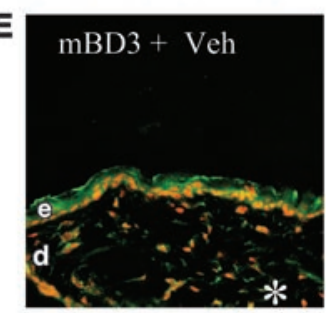

I

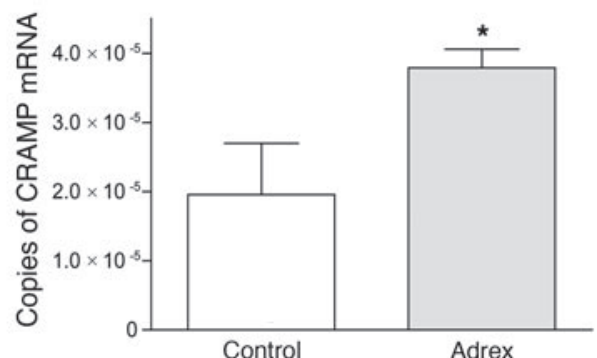

B

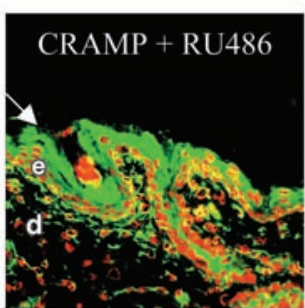

$\mathbf{F}$

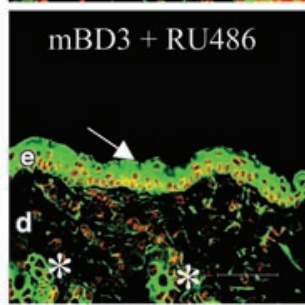

C

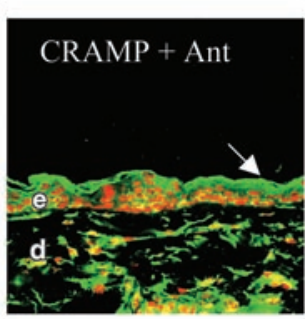

G

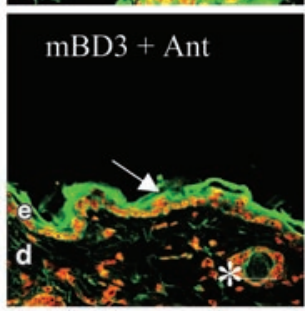

D

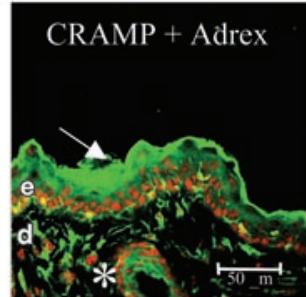

$\mathbf{H}$

mBD3 Adrex

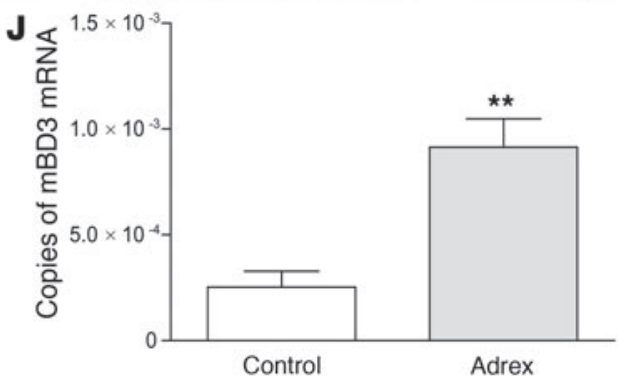

Figure 6

Endogenous GCs downregulate AMP expression in normal mouse epidermis. Immunostaining for CRAMP (A-D) and mBD3 (E-H) mRNA expression was assessed in biopsies from normal hairless mice ( $n=3$ per group) treated with RU-486 (B and $\mathbf{F})$, antalarmin $(\mathbf{C}$ and $\mathbf{G})$, or vehicle (A and $\mathbf{E}$ ) and from cohorts of adrenalectomized (adrex; D, H, I, and $\mathbf{J}$ ) and sham-operated (I and $\mathbf{J})$ hairless Skh1 mice ( $n=3$ per group). Scale bar: $50 \mu \mathrm{m}$. ${ }^{\star} P=0.04 ;{ }^{\star \star} P=0.006$.

the reported increased risk of cutaneous infections in the face of PS. Accordingly, abundant anecdotal evidence connects PS to outbreaks or progression of viral skin infections including herpes simplex, Epstein-Barr virus, HIV, influenza, and varicella/zoster virus infections $(14,16-19,21,25)$; mucosal bacterial infections such as bacterial vaginitis (51); and inflammatory dermatoses such as acne, in which microbial colonization indisputably aggravates disease expression (52). Finally, because PS exerts potent systemic effects via an increase in endogenous GCs (see below), it is highly likely that these results have implications for extracutaneous infections that occur in the face of either increased PS or systemic or topical GC therapy. Indeed, while it is generally accepted that increased endogenous GCs increase susceptibility to infection, the link to PS is more tenuous (53).

First we explored whether PS - which has well-known, adverse effects on permeability barrier homeostasis in humans and hairless mice $(29,30)$ - also adversely affects cutaneous AMP expression. In a well-characterized model of sustained PS (insomnia, crowding, and auditory stimulation in hairless mice; refs. 32, 34), we showed that PS downregulated the expression of both the murine cathelicidin CRAMP and the murine $\beta$-defensin mBD3. The fact that PS and GCs produce negative effects in human epidermis (29, $30,33)$ comparable to those in hairless mice (32-34) validates the further use of hairless mice as a model for these studies. Nevertheless, it remains possible that some of the PS-induced effects observed here could be influenced by the absence-of-function $b r$ gene product(s), because comparable studies were not performed in normal mouse skin.

We focused here on CRAMP and $\mathrm{mBD} 3$ because the human homologs of these 2 AMPs are known products of the epidermal LB secretory system $(37,38)$, and therefore, their expression could be influenced by PS or GCs, both of which reduce LB production $(33,34)$. Although the constitutive expression of these peptides is reportedly low in normal epidermis $(41,54)$, these peptides are readily detectable in normal murine epidermis by immunofluorescence, even under basal conditions, allowing us to readily discriminate further PS-induced declines in AMP protein. While PS reduced protein levels for both CRAMP and mBD3, only CRAMP declined at the mRNA level, suggesting different levels of regulation by PS for CRAMP and MBD3 (Figure 9 and see below).

We next addressed the mechanisms whereby PS adversely alters AMP expression. Because the adverse effects of PS on epidermal permeability barrier function are mediated by increased endogenous GCs $(31,32)$, we hypothesized that the effects of PS on AMP production (and on increased susceptibility to skin infections) could be attributable to increased endogenous GCs. Accordingly, both systemic and topical GCs downregulated protein levels for both CRAMP and mBD3. But systemic GCs, like PS, did not reduce mRNA levels for mBD3. Pertinently, systemic GCs also downregulate NFKB-dependent expression of at least one AMP in amphibian skin (51). The PS-endogenous GC mechanism is strongly supported by the dual observations that blockade of GC increase 


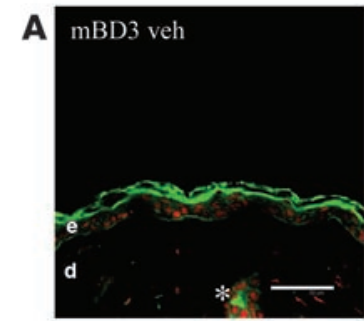

D

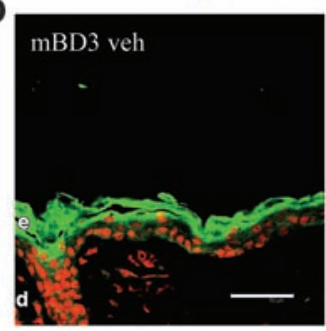

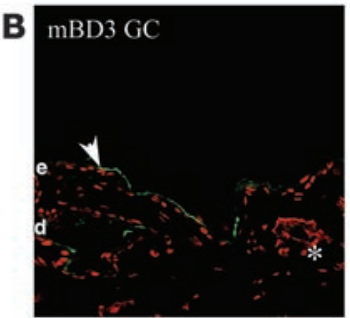

E

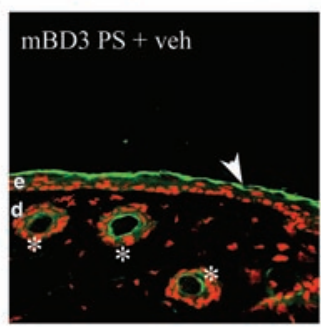

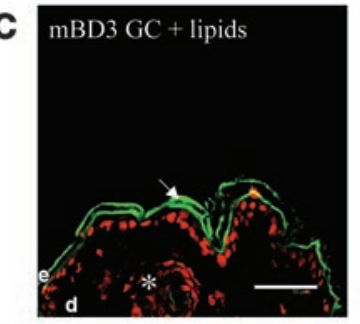

$\mathbf{F}$

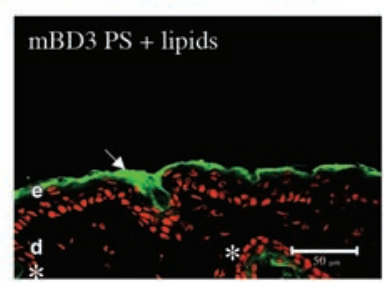

\section{Figure 7}

Coapplication of topical physiologic lipids partially normalizes mBD3 expression in the face of PS or increased GCs. Hairless mice ( $n=4$ or 5 per cohort) received either an equimolar mixture of ceramides, cholesterol, and free fatty acids (1:1:1 molar ratio; $2 \%$ final concentration) in propylene glycol/ethanol (7:3 $\mathrm{v} / \mathrm{v})$ vehicle $\left(60 \mu \mathrm{l}\right.$ to a $3-\mathrm{cm}^{2}$ area) or vehicle alone, while being cotreated with either PS ( $E$ and $\mathbf{F}$ ) or topical clobetasol (GC, B and C). Frozen sections were immunostained for mBD3 (see Methods). Controls treated only with vehicle are shown in $\mathbf{A}$ and $\mathbf{D}$. Scale bars: $50 \mu \mathrm{m}$. with systemic administration of the CRF inhibitor antalarmin and blockade of GC peripheral action with systemic administration of the GC inhibitor RU-486 both normalized AMP expression in the face of ongoing PS. The final proof of a PS-GC connection is shown by the observation that PS amplified the severity of cutaneous GAS infections, while conversely, systemic administration of RU-486 normalized resistance to this bacterial pathogen in the face of ongoing PS. Together, these studies show the important role of increased endogenous GCs in the PS-induced decline of epidermal AMP expression as well as a direct link between PS, via increased endogenous GCs, and microbial pathogenesis. Yet these studies did not address the source of the endogenous GCs that mediate the negative effects of PS on cutaneous antimicrobial defense. Increased GCs of HPA origin and/or generated within the skin (which elaborates several HP mediators, including CRF, proopiomelanocortin, and adrenocorticotropin) could, in part, account for these phenomena $(27,45,55)$. Moreover, certain cell types within skin appear capable of generating GCs $(49,50,56)$.

\section{Figure 8}

PS increases the severity of cutaneous GAS infection. (A) Female 8- to 10-wk-old Skh1/Hr mice were subjected to normal conditions or PS for $72 \mathrm{~h}$, and then subsequently injected intradermally with $4.8 \times 10^{8} \mathrm{CFU} / \mathrm{ml}$ GAS ( $n=6$ per group). Mice were then photographed daily for $4 \mathrm{~d}$ to monitor lesion size. (A) Representative lesions at day 4 from nonstressed and PS mice. (B) Lesion size (mean \pm SEM) was calculated \pm SEM for day 1 and day 4 lesions. ${ }^{*} P<0.05$ versus nonstressed. (C) Representative lesions of PS mice ( $n=5-6$ per group) immediately prior to and $72 \mathrm{~h}$ after IP injection with either vehicle or RU-486 $(6 \mathrm{mg} / \mathrm{kg})$. After $72 \mathrm{~h}$ of PS or nonstressed conditions, mice were injected intradermally with $4.8 \times 10^{8} \mathrm{CFU} /$ $\mathrm{ml}$ GAS. A representative photograph of day 4 lesions from each group is shown. (D) Lesion size (mean \pm SEM) was calculated for day 4 lesions. ${ }^{\dagger} P<0.05$ versus PS and RU-486; $" P<0.05$ versus $\mathrm{PS}$ and vehicle.
A

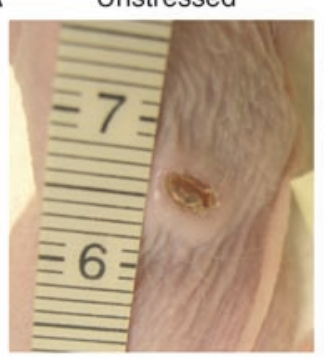

C

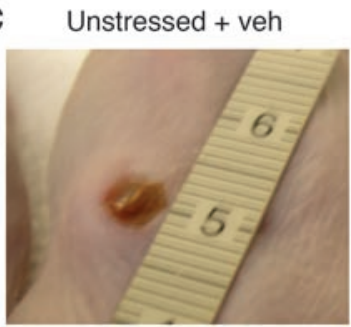

PS + veh

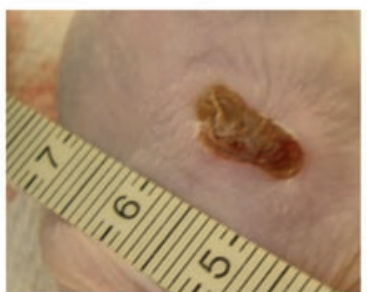

PS

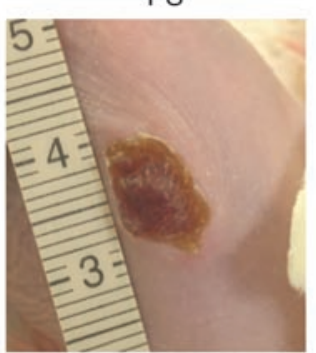

Unstressed + RU-486
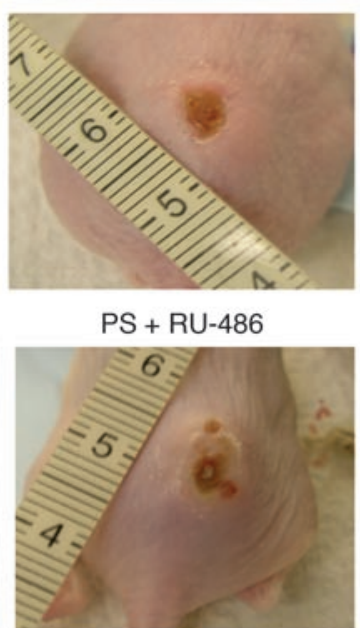

B

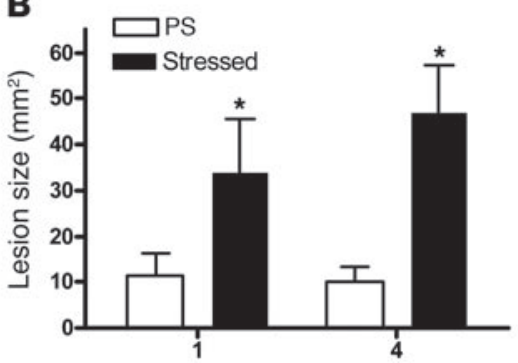

Days after infection

D

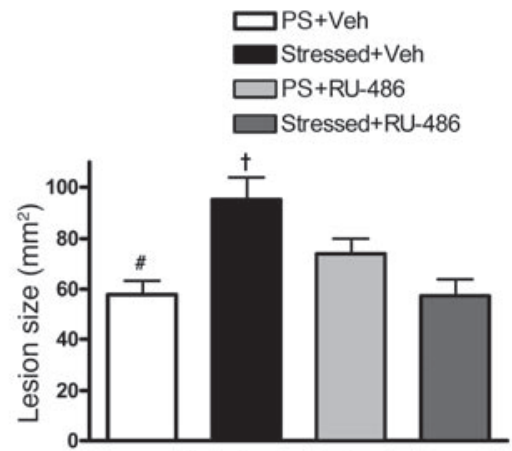

Day 4 after infection 


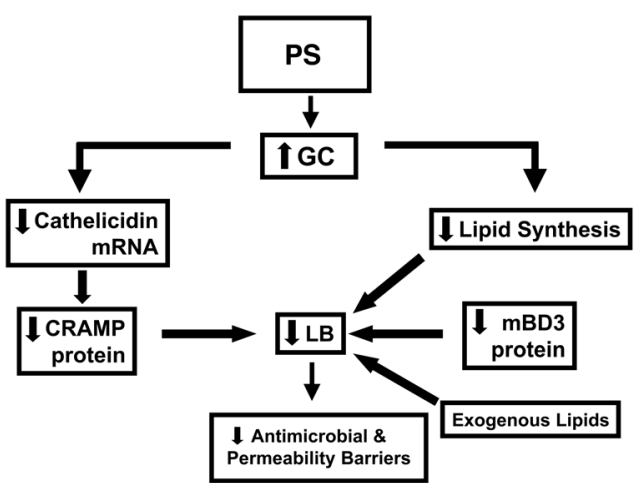

Figure 9

Divergent mechanisms for PS-induced downregulation of epidermal CRAMP and mBD3.

Thus, GCs of HPA origin and/or originating within the cutaneous neuroendocrine system could mediate the PS-induced responses described herein.

While these results provide mechanistic insights into PS-related alterations in antimicrobial defense, they also provide a likely pathomechanism for the increased risk of cutaneous (and extracutaneous) infections during PS $(13,15,18,20,24)$ as well as both systemic and topical GC therapy $(47,48,57,58)$. It should be noted, however, that PS or GC therapy could increase the risk of cutaneous infections by other, unrelated mechanisms; for example, GCs decrease epidermal dendritic cell function $(59,60)$, another important participant in cutaneous innate immunity, and also decrease epidermal primary cytokine levels (33), which could influence antimicrobial defense by a variety of downstream mechanisms. Indeed, primary cytokines regulate hBD2 expression at a transcriptional level via NFKB and AP-1 sites on the hBD2 promoter (61), and they increase hBD2 bioavailability by stimulating accumulation of nascent peptides within epidermal LB (37).

Whereas the effects of GCs (and presumably, therefore, of PS) on CRAMP protein expression can be attributed to downregulation at the mRNA level, PS and systemic GCs instead appear to decrease mBD3 expression at the protein level alone (Figure 9). The ability of topical physiologic lipids to partially override the PS-induced downregulation of $\mathrm{mBD} 3$ (whose mRNA levels do not change with PS) supports the concept that PS downregulates AMPs through increased endogenous GCs, which in turn inhibits epidermal lipid synthesis and/or LB formation (34). Yet adrenalectomized mice demonstrated an increase in $\mathrm{mBD} 3 \mathrm{mRNA}$ expression, while clobetasol treatment downregulated mRNA levels of mBD3. These divergent results can best be explained by the sustained decrease in GC production that occurs in adrenalectomized mice. Conversely, the superpotent topical steroid clobetasol likely has more profound effects on epidermal structure and metabolism than systemic GCs. Thus, the decrease in mRNA levels for mBD3 in clobetasol-treated mice likely reflects a global decrease in protein synthesis. Although the precise subcellular mechanisms whereby exogenous lipids appear to override the negative effects of PS or $\mathrm{GC}$ therapy on $\mathrm{mBD} 3$ remain uncertain, exogenous physiologic lipids have previously been shown to traverse the SC, targeting the trans-Golgi apparatus of cells in the outer nucleated layers where LBs are assembled $(62,63)$. Because packaging of protein cargo within LBs is dependent on prior or concurrent lipid deposition
(39), addition of exogenous lipids to endogenous lipid equivalents could allow additional AMPs to be loaded into nascent LBs. Our immunoelectron-microscopic results support this scheme, because exogenous lipids clearly enhanced deposition of mBD3, but not CRAMP, within LBs in the face of ongoing PS. Not only endogenous lipids (64), but also both hBD2 and the hCAP product LL-37, are copackaged within epidermal LBs $(37,38)$ and then cosecreted into the SC interstices (65). Our present results show that their murine homologs mBD3 and CRAMP were also assembled within epidermal LBs. Thus, the PS- and GC-induced downregulation of mBD3 expression appears to reflect a prior diminution in epidermal lipid synthesis and/or LB production (Figure 9).

One or more additional posttranscriptional mechanisms could contribute to the PS-induced decline in $\mathrm{mBD} 3$ protein. While physical abrogation of the epidermal permeability barrier function typically stimulates generation of IL- $1 \alpha$ and other primary cytokines $(66,67)$, topical GCs instead decrease epidermal IL- $1 \alpha$ and TNF- $\alpha$ expression (33). Thus, a PS- or GC-induced decline in cytokine signaling (33) could contribute to reduced mBD2 expression. Prior work has similarly suggested that a decline in primary cytokine production contributes to PS-induced delays in cutaneous wound healing $(9-12,22)$. Regardless of specific regulatory mechanisms, our studies suggest that exogenous physiologic lipids could benefit not only epidermal permeability barrier function in the face of PS or GCs $(33,68)$, but also cutaneous antimicrobial defense. The common effects of PS and GCs on epidermal function and AMP expression also support the putative link between the permeability and antimicrobial barriers in epidermis $(35,36)$. If operative in other epithelia, PS-induced suppression of AMPs via increased endogenous GCs could also explain the frequency and severity of infections in extraepidermal tissues of patients subjected to PS $(1-10,35,36)$. Indeed, we show here that PS downregulated AMPs in pilosebaceous structures, consistent with a microbial cause for the observation that acne vulgaris flares with increased PS (49). It is well known that PS adversely affects epithelial structure and function in gastrointestinal epithelia $(2-4,69)$; AMPs in these epithelia may be important not only for antimicrobial defense, but also for structural integrity (i.e., for the permeability barriers in several extracutaneous epithelia; refs. 69-75). Thus, it is likely, although not yet proven, that PS could abrogate AMP production in parallel with its ability to compromise extracutaneous epithelial barriers, as shown here for epidermis and pilosebaceous structures. If these speculations can be validated, systematic therapy with CRF or GCr inhibitors and/or targeted lipid replacement could improve clinical outcomes in these settings.

\section{Methods}

\section{Murine stress models}

Female hairless control mice (Skh1/Hr) and hairless Skh1 adrenalectomized mice were purchased from Charles River Laboratories and studied between 7 and $9 \mathrm{wk}$ of age. All animal experiments described in this study were conducted in accordance with accepted standards of humane animal care, under protocols approved by the local animal research committee at San Francisco VA Medical Center. Prior to beginning experiments, cohorts of 3 animals each were kept in separate cages for at least $7 \mathrm{~d}$. For the PS group, groups of 6 animals at a time ( 2 cohorts) were transferred to a 12.5 -cm-diameter, 12.5 -cm-high, transparent glass jar and exposed to continuous visible light and radio noise for $72 \mathrm{~h}$. Additional groups of PS animals were injected intraperitoneally with either RU-486 antalarmin in 
propylene glycol/ethanol $(7: 3 \mathrm{v} / \mathrm{v})$ or vehicle alone $1 \mathrm{~h}$ prior to stress onset and every $24 \mathrm{~h}$ thereafter for $72 \mathrm{~h}$. RU-486 (mifepristone; Sigma-Aldrich) and antalarmin hydrochloride (Sigma-Aldrich) were administered at doses of $6 \mathrm{mg} / \mathrm{kg}$ ( $1 \mathrm{mg} / \mathrm{ml}$ propylene glycol) and $20 \mathrm{mg} / \mathrm{kg}$, respectively. Additional mice were kept in ordinary cages ( 3 per cage), without continuous light and sound, and injected in some cases with vehicle as described above. All experimental results were replicated 2-3 times in separate experiments, and the results shown in the figures are representative of the results in replicate experiments. All mice were maintained in a temperature- and humidity-controlled room and given standard laboratory food and tap water ad libitum. There were no differences in body weights in the PS versus control groups during the course of these experiments.

\section{GC administration and blockade}

Female hairless mice (Skh1/Hr; 7-8 wk of age), as described above, were used for GC systemic and topical administration. Clobetasol $0.05 \%$ in propylene glycol/ethanol $(7: 3 \mathrm{v} / \mathrm{v})$ vehicle was applied twice daily for $3 \mathrm{~d}$ to the flanks of hairless mice. Another group of littermates received vehicle alone at the same frequency. Systemic GC was administered by injecting each mouse intraperitoneally with $9 \mu \mathrm{g}$ dexamethasone $(450 \mu \mathrm{g} / \mathrm{kg})$ in propylene glycol/ethanol $(7: 3 \mathrm{v} / \mathrm{v})$ vehicle or vehicle alone once daily for $3 \mathrm{~d}$, as described previously $(31,33)$. Dexamethasone $(10 \mathrm{nM})$ or ethanol vehicle was added to preconfluent (80\%-90\%), second-passage, cultured human keratinocytes. mRNA was harvested $18 \mathrm{~h}$ later for quantitation of hBD2 and LL-37 by RT-PCR (see below).

\section{GAS infection}

GAS infection was performed as previously described (76). Briefly, the dorsal skin of PS and nonstressed Skh1/Hr mice was injected with $50 \mu \mathrm{l}$ of a midlogarithmic growth phase $\left(\mathrm{A}_{600}=0.8 ; 4.8 \times 10^{8} \mathrm{CFU} / \mathrm{ml}\right)$ of GAS NZ131 conjugated with $50 \mu \mathrm{l}$ of sterile Cytodex beads, which acts as a carrier, or Cytodex beads alone ( $n=6$ per group). Lesions were photographed daily for $7 \mathrm{~d}$, and lesion size was quantitated in digital micrographs.

\section{Tissue preparation, protein, and RNA isolation}

Skin samples were obtained following $3 \mathrm{~d}$ of topical or systemic GC treatment, or 72 h PS with or without RU-486 or antalarmin. Epidermis from all groups was obtained by EDTA separation. Total RNA was extracted with a commercially available kit (RNeasy Mini RNA isolation kit; QIAGEN) in accordance with the manufacturer's instructions. RNA solution $(50 \mu \mathrm{l})$ was reverse-transcribed to CDNA as previously described (77). Real-time quantitative PCR was performed on an ABI 7900 machine using SYBR Green detection as previously described for the $\mathrm{mBD} 3$, murine cathelicidin, and $18 \mathrm{~S}$ RNA (internal control) measurements $(40,41)$. The SYBR Green kit was purchased from Applied Biosystems. Primer sequences were: mBD3 forward, ATTTCTCCTGGTGCTCGTGT; reverse, GGAACTCCACAACTGCCAAT; 18S RNA forward, GTAACCCGTTGAACCCCATT; reverse, CCATCCAATCGGTAGTAGCG. The mBD3 and 18S RNA PCR primers were synthesized by Biosearch Technologies. Standard reaction volumes were $20 \mu \mathrm{l}$ with $250 \mathrm{ng}$ cDNA and $125 \mathrm{nM} \mathrm{hBD} 2$ and mBD3, $500 \mathrm{nM} 18 \mathrm{~S}$ $\mathrm{RNA}$, or $1 \mu \mathrm{M}$ final concentration of the cathelicidin primers. Initial steps of RT-PCR were $2 \mathrm{~min}$ at $50^{\circ} \mathrm{C}, 10$-min hold at $95^{\circ} \mathrm{C}$, and 40 cycles of a 15 -s melt at $95^{\circ} \mathrm{C}$ followed by a 300 -s annealing/extension at $60^{\circ} \mathrm{C}$. All reactions were performed in triplicate. The threshold for threshold cycle $(\mathrm{Ct})$ analysis of all samples was set at 0.50 relative fluorescence units. Quantitative PCR data were analyzed using the $2^{-\Delta \Delta \mathrm{Ct}}$ method $(78,79)$.

\section{Western immunoblotting}

For Western blotting, a method was employed for extraction of low-molecular weight, hydrophobic, cationic peptides, as described previously (80).
Briefly, epidermis was separated from mouse skin after incubations of full-thickness pieces of flank skin in $10 \mathrm{mM}$ EDTA in PBS at $37^{\circ} \mathrm{C}$ for $2 \mathrm{~h}$. Extractions were performed in an acidic buffer (30\% acetonitrile, $0.1 \%$ formic acid; $\mathrm{pH}<3$ ); extracts were homogenized on ice and centrifuged at $17,530 \mathrm{~g}$ for $30 \mathrm{~min}$ at $4^{\circ} \mathrm{C}$, and then the supernatants were re-centrifuged for $15 \mathrm{~min}$ prior to protein fractionation. An equal amount of extracted protein in NuPAGE sample buffer in water was heated at $85^{\circ} \mathrm{C}$, without reducing agents, followed by loading of equal amounts of samples from each experimental group onto $10 \%$ tricine gels (Invitrogen), using $\beta$-actin as an internal standard. After electrophoresis, proteins were transferred from gels onto PVDF membranes and electrophoresed for $1 \mathrm{~h}$ in tricine/ glycine transfer buffer, followed by immunoblotting with the rabbit antimouse mBD3 antibody (Alpha Diagnostics). Antibody binding to mBD3 was detected with the Western-Breeze chemiluminescence kit, following the manufacturer's protocol (Invitrogen).

\section{Morphologic studies}

Immunofluorescence. Skin biopsy samples were taken ( $n=3$ per group) and snap-frozen in liquid nitrogen in a tissue-embedding medium. Frozen sections $(8 \mu \mathrm{m})$ were soaked in acetone for $10 \mathrm{~min}$, washed in PBS, and blocked with $4 \%$ BSA and $0.05 \%$ cold fish gelatin in PBS for $30 \mathrm{~min}$. Slides were then incubated overnight at $4^{\circ} \mathrm{C}$ with CRAMP or mBD3 (Alpha Diagnostic) primary antibodies, followed by incubation with FITC-conjugated goat anti-rabbit secondary antibody for $40 \mathrm{~min}$ at room temperature. Slides were counterstained with propidium iodide and visualized on a Leica TCS-SP confocal microscope. Controls without primary antibodies showed no immunolabeling, and skin sections of CRAMP knockout mice served as controls.

Electron microscopy. Skin biopsies from PS and nonstressed mice were minced to less than $0.5 \mathrm{~mm}^{3}$, fixed in modified Karnovsky's fixative overnight, and postfixed in $0.5 \% \mathrm{RuO}_{4}$ and $2 \% \mathrm{OsO}_{4}$ containing $1.5 \%$ potassium ferrocyanide (81). After postfixation, all samples were dehydrated in graded ethanol solutions and embedded in an Epon-epoxy mixture. Ultrathin sections were examined, with or without further lead citrate contrasting, using a Zeiss $10 \mathrm{~A}$ electron microscope operated at $60 \mathrm{kV}$.

Immunoelectron microscopy. Skin biopsies for immunoelectron microscopy were cut into $0.5-\mathrm{mm}^{3}$ pieces and microwave-fixed for $2.5 \mathrm{~min}$ in $0.1 \mathrm{M}$ cacodylate buffer ( $\mathrm{pH} 7.4$, containing $0.1 \%$ sodium periodate, $0.6 \%$ L-lysine, and $\mathrm{CaCl}_{2}$ ) at $37^{\circ} \mathrm{C}$. Samples were postfixed in reduced osmium tetroxide $\left(2 \% \mathrm{OsO}_{4}\right.$ and $1.5 \%$ potassium ferrocyanide in $0.1 \mathrm{M}$ cacodylate buffer, $\mathrm{pH} 7.4$ ) for $2.5 \mathrm{~min}$ in the microwave at $37^{\circ} \mathrm{C}$. Samples were then washed 3 times in double-distilled water; postfixed for $1 \mathrm{~h}$ in $2 \%$ aqueous uranyl acetate at $4{ }^{\circ} \mathrm{C}$; dehydrated in graded ethanol solutions; transferred first to a 1:1, then a $2: 1$, mixture of LR White resin (EMS) in $100 \%$ ethanol for 15 min each; and finally infiltrated in $100 \%$ LR White for $2 \mathrm{~h}$ (all steps at $4^{\circ} \mathrm{C}$ ). Polymerization was carried out at $40^{\circ} \mathrm{C}$ under a vacuum for $2 \mathrm{~d}$. Ultrathin sections were collected on Formvar-coated nickel grids.

All immunolabeling steps were carried out at room temperature. Nonspecific binding sites were blocked with $5 \%$ BSA, $5 \%$ normal goat serum, and $0.1 \%$ cold-water fish gelatin in PBS for $20 \mathrm{~min}$, after which grids were washed 3 times for 5 min each in incubation buffer (0.1\% Aurion BSA-c; EMS; in PBS). Primary antibodies (rabbit anti-CRAMP and rabbit anti$\mathrm{mBD} 3$ ) were used at a 1:10 dilution in incubation buffer for $1 \mathrm{~h}$. After 3 washes for 5 min each with incubation buffer, sections were incubated with goat anti-rabbit IgG, conjugated to $10 \mathrm{~nm}$ colloidal gold (Ted Pella Inc.) for $1 \mathrm{~h}$. After secondary immunolabeling, grids were washed 6 times with incubation buffer followed by 3 washes with PBS, fixed in $2 \%$ glutaraldehyde in PBS for $5 \mathrm{~min}$, followed by a final water wash for $30 \mathrm{~s}$. Sections were examined by electron microscopy after contrasting with both $2 \%$ aqueous uranyl acetate and $0.6 \%$ lead citrate. Quantitation 
of immunolabeling was carried out in coded, randomized micrographs $(n \geq 10$ each) from 4 different samples.

\section{Statistics}

Data were expressed as mean \pm SEM. Statistical analyses were performed using paired and unpaired Student's $t$ tests. A $P$ value less than 0.05 was considered significant.

\section{Acknowledgments}

These studies were supported by NIH grants AR19098, AR39448(PP), and AI059311 and by the Medical Research Service, Department of Veterans Affairs. K.M. Aberg was supported by an NIH postdoctoral fellowship (AR07175-28). Jens Schröder (University of Kiel, Kiel, Germany) provided invaluable advice in preparing samples for Western blotting. We gratefully acknowledge the excellent editorial assistance of Joan Wakefield and Jerelyn Magnusson.

Received for publication February 6, 2007, and accepted in revised form July 16, 2007.

Address correspondence to: Peter M. Elias, Dermatology Service (190), VA Medical Center, 4150 Clement Street, San Francisco, California 94121, USA. Phone: (415) 750-2091; Fax: (415) 751-3927; E-mail: eliasp@derm.ucsf.edu.

Portions of this work were presented at the annual meeting of the Society for Investigative Dermatology in Philadelphia, Pennsylvania, USA, May 3-6, 2006, and published in abstract form.
1. Glaser, R. 2005. Stress-associated immune dysregulation and its importance for human health: a personal history of psychoneuroimmunology. Brain Behav. Immun. 19:3-11.

2. Meddings, J.B., and Swain, M.G. 2000. Environmental stress-induced gastrointestinal permeability is mediated by endogenous glucocorticoids in the rat. Gastroenterology. 119:1019-1028.

3. Mawdsley, J.E., and Rampton, D.S. 2005. Psychological stress in IBD: new insights into pathogenic and therapeutic implications. Gut. 54:1481-1491.

4. Jones, M.P. 2006. The role of psychosocial factors in peptic ulcer disease: beyond Helicobacter pylori and NSAIDs. J. Psychosom. Res. 60:407-412.

5. Strike, P.C., and Steptoe, A. 2004. Psychosocial factors in the development of coronary artery disease. Prog. Cardiovasc. Dis. 46:337-347.

6. Rozanski, A., Blumenthal, J.A., Davidson, K.W., Saab, P.G., and Kubzansky, L. 2005. The epidemiology, pathophysiology, and management of psychosocial risk factors in cardiac practice: the emerging field of behavioral cardiology. J. Am. Coll. Cardiol. 45:637-651.

7. Marucha, P.T., Kiecolt-Glaser, J.K., and Favagehi, M. 1998. Mucosal wound healing is impaired by examination stress. Psychosom. Med. 60:362-365.

8. Spiegel, D. 1999. Healing words: emotional expression and disease outcome. JAMA. 281:1328-1329.

9. Broadbent, E., Petrie, K.J., Alley, P.G., and Booth, R.J. 2003. Psychological stress impairs early wound repair following surgery. Psychosom. Med. 65:865-869.

10. Ebrecht, M., et al. 2004. Perceived stress and cortisol levels predict speed of wound healing in healthy male adults. Psychoneuroendocrinology. 29:798-809.

11. Horan, M.P., et al. 2005. Impaired wound contraction and delayed myofibroblast differentiation in restraint-stressed mice. Brain Behav. Immun. 19:207-216.

12. Kiecolt-Glaser, J.K., et al. 2005. Hostile marital interactions, proinflammatory cytokine production, and wound healing. Arch. Gen. Psychiatry. 62:1377-1384

13. Ben-Nathan, D., Lustig, S., and Feuerstein, G. 1989. The influence of cold or isolation stress on neuroinvasiveness and virulence of an attenuated variant of West Nile virus. Arch. Virol. 109:1-10.

14. Rein, M. 2000. Stress and genital herpes recurrences in women. JAMA. 283:1394.

15. Mohren, D.C., Swaen, G.M., van Amelsvoort, L.G. Borm, P.J., and Galama, J.M. 2003. Job insecurity as a risk factor for common infections and health complaints. J. Occup. Environ. Med. 45:123-129.

16. Ortiz, G.C., Sheridan, J.F., and Marucha, P.T. 2003. Stress-induced changes in pathophysiology and interferon gene expression during primary HSV-1 infection. Brain Behav. Immun. 17:329-338.

17. Pereira, D.B., et al. 2003. Stress as a predictor of symptomatic genital herpes virus recurrence in women with human immunodeficiency virus.

\section{J. Psychosom. Res. 54:237-244.}

18. Hunzeker, J., Padgett, D.A., Sheridan, P.A., Dhabhar, F.S., and Sheridan, J.F. 2004. Modulation of natural killer cell activity by restraint stress during an influenza A/PR8 infection in mice. Brain Behav. Immun. 18:526-535.

19. Kopnisky, K.L., Stoff, D.M., and Rausch, D.M. 2004. Workshop report: the effects of psychological variables on the progression of HIV-1 disease. Brain Behav. Immun. 18:246-261.

20. Gomez-Merino, D., et al. 2005. Effects of combined stress during intense training on cellular immunity, hormones and respiratory infections. Neuroimmunomodulation. 12:164-172.

21. Johnson, R.R., et al. 2006. Social stress alters the severity and onset of the chronic phase of Theiler's virus infection. J. Neuroimmunol. 175:39-51.

22. Glaser, R., et al. 1999. Stress-related changes in proinflammatory cytokine production in wounds. Arch. Gen. Psychiatry. 56:450-456.

23. Brogden, K.A., Guthmiller, J.M., Salzet, M., and Zasloff, M. 2005. The nervous system and innate immunity: the neuropeptide connection. Nat. Immunol. 6:558-564.

24. Kiank, C., et al. 2006. Stress susceptibility predicts the severity of immune depression and the failure to combat bacterial infections in chronically stressed mice. Brain Behav. Immun. 20:359-368.

25. Cacioppo, J.T., et al. 2002. Autonomic and glucocorticoid associations with the steady-state expression of latent Epstein-Barr virus. Horm. Behav. 42:32-41.

26. Slominski, A.T., et al. 1999. Cutaneous expression of CRH and CRH-R. Is there a "skin stress response system?” Ann. N. Y. Acad. Sci. 885:287-311.

27. Slominski, A., Wortsman, J., Luger, T., Paus, R., and Solomon, S. 2000. Corticotropin releasing hormone and proopiomelanocortin involvement in the cutaneous response to stress. Physiol. Rev. 80:979-1020.

28. Denda, M., Tsuchiya, T., Hosoi, J., and Koyama, J. 1998. Immobilization-induced and crowded environment-induced stress delay barrier recovery in murine skin. Br. J. Dermatol. 138:780-785.

29. Altemus, M., Rao, B., Dhabhar, F.S., Ding, W., and Granstein, R.D. 2001. Stress-induced changes in skin barrier function in healthy women. J. Invest. Dermatol. 117:309-317.

30. Garg, A., et al. 2001. Psychological stress perturbs epidermal permeability barrier homeostasis: implications for the pathogenesis of stress-associated skin disorders. Arch. Dermatol. 137:53-59.

31. Denda, M., Tsuchiya, T., Elias, P.M., and Feingold, K.R. 2000. Stress alters cutaneous permeability barrier homeostasis. Am. J. Physiol. Regul. Integr. Comp. Physiol. 278:R367-R372.

32. Choi, E.H., et al. 2006. Glucocorticoid blockade reverses psychological stress-induced abnormalities in epidermal structure and function. Am. J. Physiol.
Regul. Integr. Comp. Physiol. 291:R1657-R1662.

33. Kao, J.S., et al. 2003. Short-term glucocorticoid treatment compromises both permeability barrier homeostasis and stratum corneum integrity: inhibition of epidermal lipid synthesis accounts for functional abnormalities. J. Invest. Dermatol. 120:456-464.

34. Choi, E.H., et al. 2005. Mechanisms by which psychologic stress alters cutaneous permeability barrier homeostasis and stratum corneum integrity. J. Invest. Dermatol. 124:587-595.

35. Elias, P.M., and Choi, E.H. 2005. Interactions among stratum corneum defensive functions. Exp. Dermatol. 14:719-726.

36. Elias, P.M. 2005. Stratum corneum defensive functions: an integrated view. J. Invest. Dermatol. 125:183-200.

37. Oren, A., Ganz, T., Liu, L., and Meerloo, T. 2003. In human epidermis, beta-defensin 2 is packaged in lamellar bodies. Exp. Mol. Pathol. 74:180-182.

38. Braff, M.H., Di Nardo, A., and Gallo, R.L. 2005. Keratinocytes store the antimicrobial peptide cathelicidin in lamellar bodies. J. Invest. Dermatol. 124:394-400.

39. Rassner, U., Feingold, K.R., Crumrine, D.A., and Elias, P.M. 1999. Coordinate assembly of lipids and enzyme proteins into epidermal lamellar bodies. Tissue Cell. 31:489-498.

40. Ganz, T. 2003. Defensins: antimicrobial peptides of innate immunity. Nat. Rev. Immunol. 3:710-720.

41. Braff, M.H., and Gallo, R.L. 2006. Antimicrobial peptides: an essential component of the skin defensive barrier. Curr. Top. Microbiol. Immunol. 306:91-110.

42. Nizet, V., et al. 2001. Innate antimicrobial peptide protects the skin from invasive bacterial infection. Nature. 414:454-457.

43. O’Sullivan, R.L., Lipper, G., and Lerner, E.A. 1998. The neuro-immuno-cutaneous-endocrine network: relationship of mind and skin. Arch. Dermatol. 134:1431-1435.

44. Paus, R., Theoharides, T.C., and Arck, P.C. 2006. Neuroimmunoendocrine circuitry of the 'brainskin connection'. Trends Immunol. 27:32-39.

45. Slominski, A., et al. 2006. Corticotropin releasing hormone and the skin. Front. Biosci. 11:2230-2248.

46. Ito, N., et al. 2005. Human hair follicles display a functional equivalent of the hypothalamic-pituitary-adrenal axis and synthesize cortisol. FASEB J. 19:1332-1334.

47. Hengge, U.R., Ruzicka, T., Schwartz, R.A., and Cork, M.J. 2006. Adverse effects of topical glucocorticosteroids. J. Am. Acad. Dermatol. 54:1-15; quiz 16-18.

48. Lionakis, M.S., and Kontoyiannis, D.P. 2003. Glucocorticoids and invasive fungal infections. Lancet. 362:1828-1838.

49. Slominski, A., Zbytek, B., Semak, I., Sweatman, T., and Wortsman, J. 2005. CRH stimulates POMC activity and corticosterone production in dermal 
fibroblasts. J. Neuroimmunol. 162:97-102.

50. Slominski, A., et al. 2005. CRH stimulation of corticosteroids production in melanocytes is mediated by ACTH. Am. J. Physiol. Endocrinol. Metab. 288:E701-E706

51. Simmaco, M., et al. 1997. Effect of glucocorticoids on the synthesis of antimicrobial peptides in amphibian skin. FEBS Lett. 416:273-275.

52. Chiu, A., Chon, S.Y., and Kimball, A.B. 2003. The response of skin disease to stress: changes in the severity of acne vulgaris as affected by examination stress. Arch. Dermatol. 139:897-900.

53. Culhane, J.F., Rauh, V., McCollum, K.F., Elo, I.T., and Hogan, V. 2002. Exposure to chronic stress and ethnic differences in rates of bacterial vaginosis among pregnant women. Am. J. Obstet. Gynecol. 187:1272-1276.

54. de Jongh, G.J., et al. 2005. High expression levels of keratinocyte antimicrobial proteins in psoriasis compared with atopic dermatitis. J. Invest. Dermatol. 125:1163-1173.

55. Slominski, A., Wortsman, J., Tuckey, R.C., and Paus, R. 2007. Differential expression of HPA axis homolog in the skin. Mol. Cell. Endocrinol. 265-266:143-149.

56. Slominski, A., et al. 2004. A novel pathway for sequential transformation of 7-dehydrocholesterol and expression of the P450scc system in mammalian skin. Eur. J. Biochem. 271:4178-4188.

57. Kang, I., and Park, S.H. 2003. Infectious complications in SLE after immunosuppressive therapies. Curr. Opin. Rheumatol. 15:528-534.

58. Encke, J., Uhl, W., Stremmel, W., and Sauer, P. 2004. Immunosuppression and modulation in liver transplantation. Nephrol. Dial. Transplant. 19(Suppl. 4):iv22-iv25.

59. Hoetzenecker, W., et al. 2004. Corticosteroids but not pimecrolimus affect viability, maturation and immune function of murine epidermal Langerhans cells. J. Invest. Dermatol. 122:673-684.

60. Krummen, M.B., et al. 2006. Effect of pimecrolimus vs. corticosteroids on murine bone marrow-derived dendritic cell differentiation, maturation and func- tion. Exp. Dermatol. 15:43-50.

61. Wehkamp, K., Schwichtenberg, L., Schroder, J.M., and Harder, J. 2006. Pseudomonas aeruginosa- and IL-1beta-mediated induction of human beta-defensin-2 in keratinocytes is controlled by NF-kappaB and AP-1. J. Invest. Dermatol. 126:121-127.

62. Mao-Qiang, M., Feingold, K.R., and Elias, P.M. 1993. Exogenous lipids influence permeability barrier recovery in acetone-treated murine skin. Arch. Dermatol. 129:728-738.

63. Mao-Qiang, M., Brown, B.E., Wu-Pong, S., Feingold, K.R., and Elias, P.M. 1995. Exogenous nonphysiologic vs physiologic lipids. Divergent mechanisms for correction of permeability barrier dysfunction. Arch. Dermatol. 131:809-816.

64. Feingold, K.R. 1991. The regulation and role of epidermal lipid synthesis. Adv. Lipid Res. 24:57-82.

65. Huh, W.K., et al. 2002. Dynamic alteration of human beta-defensin 2 localization from cytoplasm to intercellular space in psoriatic skin. J. Mol. Med. 80:678-684.

66. Wood, L.C., Jackson, S.M., Elias, P.M., Grunfeld, C., and Feingold, K.R. 1992. Cutaneous barrier perturbation stimulates cytokine production in the epidermis of mice. J. Clin. Invest. 90:482-487.

67. Nickoloff, B.J., and Naidu, Y. 1994. Perturbation of epidermal barrier function correlates with initiation of cytokine cascade in human skin. J. Am. Acad. Dermatol. 30:535-546.

68. Man, M.Q., et al. 2006. Basis for improved permeability barrier homeostasis induced by PPAR and LXR activators: liposensors stimulate lipid synthesis, lamellar body secretion, and post-secretory lipid processing. J. Invest. Dermatol. 126:386-392.

69. Levy, R.L., et al. 2006. Psychosocial aspects of the functional gastrointestinal disorders. Gastroenterology. 130:1447-1458.

70. Taupin, D., and Podolsky, D.K. 2003. Trefoil factors: initiators of mucosal healing. Nat. Rev. Mol. Cell Biol. 4:721-732.

71. Cameron, H.L., and Perdue, M.H. 2005. Stress impairs murine intestinal barrier function: improvement by glucagon-like peptide-2. J. Pharmacol. Exp. Ther. 314:214-220.

72. Shaykhiev, R., et al. 2005. Human endogenous antibiotic LL-37 stimulates airway epithelial cell proliferation and wound closure. Am. J. Physiol. Lung Cell. Mol. Physiol. 289:L842-L848.

73. Yang, Y.H., et al. 2006. The cationic host defense peptide rCRAMP promotes gastric ulcer healing in rats. J. Pharmacol. Exp. Ther. 318:547-554.

74. Heilborn, J.D., et al. 2003. The cathelicidin antimicrobial peptide LL-37 is involved in re-epithelialization of human skin wounds and is lacking in chronic ulcer epithelium. J. Invest. Dermatol. 120:379-389.

75. Strober, W. 2006. Immunology. Unraveling gut inflammation. Science. 313:1052-1054.

76. Betschel, S.D., Borgia, S.M., Barg, N.L., Low, D.E., and De Azavedo, J.C. 1998. Reduced virulence of group A streptococcal Tn916 mutants that do not produce streptolysin S. Infect. Immun. 66:1671-1679.

77. Tirmenstein, M.A., Nicholls-Grzemski, F.A., Schmittgen, T.D., Zakrajsek, B.A., and Fariss, M.W. 2000. Characterization of nitric oxide production following isolation of rat hepatocytes. Toxicol. Sci. 53:56-62.

78. Winer, J., Jung, C.K., Shackel, I., and Williams, P.M. 1999. Development and validation of real-time quantitative reverse transcriptase-polymerase chain reaction for monitoring gene expression in cardiac myocytes in vitro. Anal. Biochem. 270:41-49.

79. Schmittgen, T.D., et al. 2000. Quantitative reverse transcription-polymerase chain reaction to study mRNA decay: comparison of endpoint and realtime methods. Anal. Biochem. 285:194-204.

80. Schroder, J.M. 2001. Isolation and purification of chemokines from natural sources. Mol. Biotechnol. 18:71-77.

81. Hou, S.Y., et al. 1991. Membrane structures in normal and essential fatty acid-deficient stratum corneum: characterization by ruthenium tetroxide staining and $\mathrm{x}$-ray diffraction. J. Invest. Dermatol. 96:215-223. 